\title{
Ex vivo and in vivo coronary ostial locations in humans
}

\section{Journal Article}

\section{Author(s):}

Knight, Joseph; Kurtcuoglu, Vartan; Muffly, Karl; Marshall, William; Stolzmann, Paul; Desbiolles, Lotus; Seifert, Burkhardt; Poulikakos, Dimos; Alkadhi, Hatem

\section{Publication date:}

2009-10

\section{Permanent link:}

https://doi.org/10.3929/ethz-b-000016375

\section{Rights / license:}

In Copyright - Non-Commercial Use Permitted

\section{Originally published in:}

Surgical and radiologic anatomy 31(8), https://doi.org/10.1007/s00276-009-0488-9 


\title{
Ex vivo and in vivo coronary ostial locations in humans
}

\author{
Joseph Knight • Vartan Kurtcuoglu • Karl Muffly • \\ William Marshall Jr · Paul Stolzmann · Lotus Desbiolles • \\ Burkhardt Seifert · Dimos Poulikakos · Hatem Alkadhi
}

Received: 24 July 2008 / Accepted: 23 February 2009 / Published online: 14 March 2009

(C) Springer-Verlag 2009

\begin{abstract}
Purpose Knowledge of the normal in vivo distribution and variation of coronary ostial locations is essential in the planning of various interventional and surgical procedures. However, all studies to date have reported the distribution of coronary ostia locations only in cadaver hearts. In this study, we sought to assess the distribution of coronary ostial locations in patients using cardiac dual-source computed tomography (CT) and compare these values to those of human cadaveric specimens.

Methods Measurements of the coronary ostia location were performed in 150 patients undergoing dual-source CT and in 75 cadavers using open measurement techniques. All 150 patients had a normal aortic valve function and no previous cardiac intervention or surgery. The location of the right and left coronary origin in relation to the aortic annulus and the height of the sinus of Valsalva were measured.
\end{abstract}

J. Knight · V. Kurtcuoglu $\cdot$ D. Poulikakos $(\varangle)$ Laboratory of Thermodynamics in Emerging Technologies, Department of Mechanical and Process Engineering, ETH Zurich, Sonneggstrasse 3, ML J 36, 8092 Zurich, Switzerland e-mail: dimos.poulikakos@ethz.ch

K. Muffly · W. Marshall Jr

College of Medicine, University of South Florida,

Tampa, FL, USA

P. Stolzmann · L. Desbiolles · H. Alkadhi

Biostatistics Unit, ISPM, University of Zurich,

Zurich, Switzerland

B. Seifert

Institute of Diagnostic Radiology,

University Hospital Zurich, Zurich, Switzerland
Results Mean ostial locations at CT were $17.0( \pm 3.6) \mathrm{mm}$ and $15.3( \pm 3.1) \mathrm{mm}$ for the right and left coronary ostia, with large variations of both sides (right: $10.4-28.5 \mathrm{~mm}$; left: $9.8-29.3 \mathrm{~mm}$ ). In cadavers, mean locations were 14.9 $( \pm 4.3) \mathrm{mm}[5-24 \mathrm{~mm}]$ for right and $16.0( \pm 3.6) \mathrm{mm}[9-$ $24 \mathrm{~mm}$ ] for left coronary ostia. Comparison of CT and cadaver data showed statistically significant differences for right $(P<0.0001)$ but not left $(P=0.1675)$ coronary ostia.

Conclusions This study provides data of normal coronary ostial origins and demonstrates significant differences between in vivo and ex vivo measurements regarding the right coronary ostium. The observed large variations of coronary ostia origins emphasize the importance of considering such anatomic variations in the development of treatments.

Keywords Coronary ostia $\cdot$ Coronary artery $\cdot$ Heart . Anatomy $\cdot$ Computed tomography

\section{Introduction}

Accurate knowledge of the locations of the coronary ostia in relation to the aortic root is critical for a number of interventional and surgical cardiovascular procedures, including cannulation or catheterization of the coronary arteries, aortic graft repair or root replacement, and implantation of percutaneous aortic valves (PAV) or transapical valve replacement. The recent advent of PAV, providing a non-operative treatment of symptomatic aortic valve disease, has necessitated continuing developments of devices, techniques, and treatment protocols for optimization of PAV procedures [14]. With the proximity of the coronary ostia to the aortic annulus and valve leaflets, a particularly challenging issue is the 
risk of obstruction of the coronary ostia during PAV replacement $[2,4,9,11]$.

In spite of the advances in treatment of aortic valve and root pathology, an in vivo data set on the variation of the normal coronary ostial location in patients is not available. Though some studies $[6,12,15,21,22]$ have been performed to determine ostial locations (Table 1), each of these studies was limited in that the measurements were taken from cadavers. Further, challenges associated with determining the exact location of the reference points for making such measurements can be seen in the fact that each previous study uses a different method of measurement. Though the ability to make such measurements more precisely using computed tomography (CT) or magnetic resonance imaging (MRI), which allows accurate measurements of three-dimensional cardiac structures, has been foreseen for many years, it was only recently that spatial and temporal resolution was sufficient to do so. Recent developments in CT technology provide a high temporal resolution in combination with an isotropic spatial resolution which allows virtually motion artifact-free imaging of the heart and coronary arteries in any arbitrary three-dimensional plane [16].

In this study we sought to provide data sets representing the normal distribution of the coronary ostial locations relative to the aortic root anatomy in a cohort of consecutive patients undergoing CT imaging of the heart and aortic root. Given the varied methods of measurements of the ostial location of previous ex vivo studies $[6,12,15,21$, 22], we also took measurements of the ostial locations in a sample of cadavers to determine the variance of ex vivo measurements to those taken in vivo. We sought to identify to what extent the in vivo measurements of the coronary ostial locations differ from those made in cadavers.

\section{Materials and methods}

\section{Cadavers}

Seventy-five adult hearts (sex not available) explanted from human cadavers $(\geq 40 \mathrm{y} / \mathrm{o})$ were studied by a single observer (J.K.). Right and left coronary ostial locations for each of these hearts were recorded using a standard engineering caliper. In all cadavers, the locations of the coronary ostia were taken as the distance measured orthogonally from the aortic annulus (at the junction of the sinus of Valsalva and the aortic cusps), superior and perpendicular to the center of the coronary ostium (Fig. 1a). Particular attention was paid to ensure that the lower reference point of the junction of the sinus of Valsalva to the aortic cusp was accurately obtained.

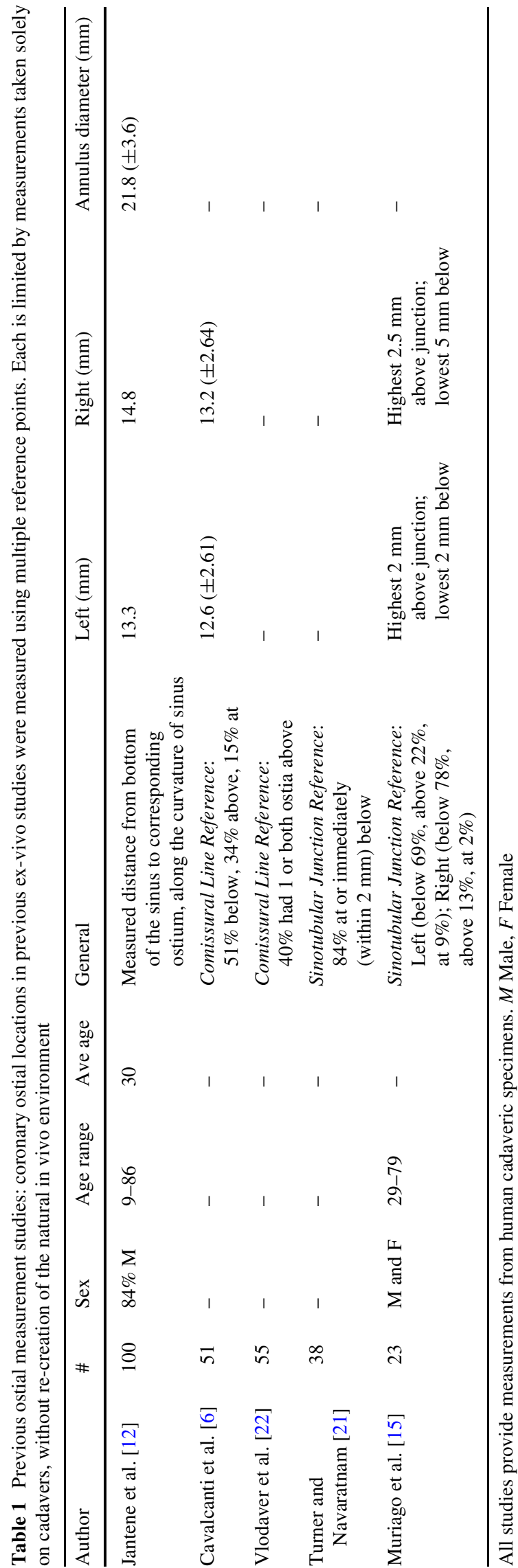




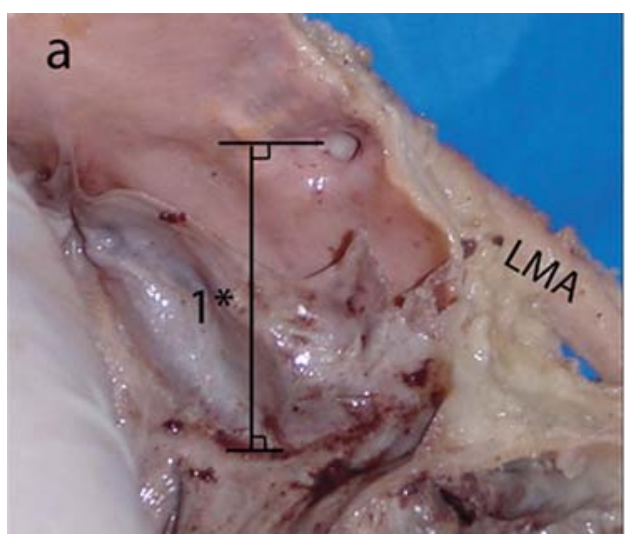

Fig. 1 Coronary ostial and sinus measurements-Measurements of the coronary ostial locations in cadavers (a) and with CT (b) were made from the base of the aortic annulus perpendicularly up to the center of the coronary ostium. $1=$ Measurement for the left main artery (LMA) ostium in cadavers (a) and in patients with CT (b); $4=$ Measurement for the right coronary artery (RCA) ostium with CT.

\section{Patients}

One-hundred fifty consecutive Caucasian patients (mean age $60.4 \pm 12.0$; range $29-86$ years) undergoing cardiac CT from July 2006 to April 2007 were included in this study. Demographic details of the patient population can be found in Table 2. Mean height, weight, and body surface area [8] (BSA) were $169.5( \pm 9.6) \mathrm{cm}, 74.0( \pm 14.0) \mathrm{kg}$, and $1.82( \pm 0.17) \mathrm{m}^{2}$, respectively. There were $58(38.7 \%)$ females (mean age $60.5 \pm 12.3$, range 29-82 years) and 92 (61.3\%) males (mean age $60.5 \pm 11.8$, range 33-86 years, $P=0.49$ ). BSA for the females was $1.70 \pm 0.16 \mathrm{~m}^{2}$, while $\mathrm{BSA}$ in the male group was $1.93 \pm 0.14 \mathrm{~m}^{2}(P<0.0001$ versus female BSA). The majority of the patients $(84 \%)$ had a low pre-test likelihood of CAD, whereas an intermediate likelihood was found in $16 \%$ of the patients [7]. Indications for cardiac CT were in accordance with recent recommendations $[5,10]$ : atypical chest pain in combination with a low to intermediate risk for coronary artery

Table 2 Demographic data: CT patient group demographic information

\begin{tabular}{|c|c|}
\hline Demographic & Value \\
\hline Age & $60.4 \pm 12.0$ \\
\hline Gender & $61.3 \%$ Male \\
\hline Height & $169.5( \pm 9.6) \mathrm{cm}$ \\
\hline Weight & $74.0( \pm 14.0) \mathrm{kg}$ \\
\hline BSA & $1.82( \pm 0.17) \mathrm{m}^{2}$ \\
\hline \multicolumn{2}{|c|}{ Pre-test likelihood of CAD } \\
\hline Low & $84 \%$ \\
\hline Intermediate & $16 \%$ \\
\hline
\end{tabular}

$B S A$ Body surface area, $C A D$ Coronary artery disease

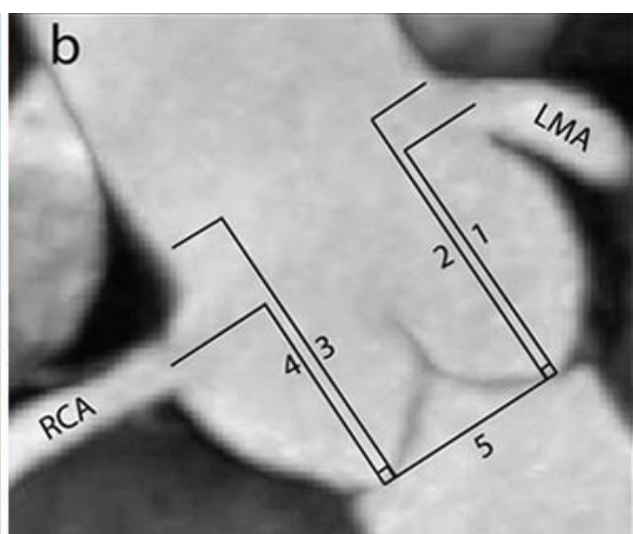

Measurements of the left (2) and right (3) sinus heights were measured by moving through the planes to obtain the attachment of the sinus at the aortic annulus and the sino-tubular junction (yellow dashed line represents the sinus shape through multiple planes). $5=$ measurement of the aortic annulus with CT (b)

disease $(n=111)$, patients prior to extra-cardiac surgery for preoperative evaluation of their coronary arteries (also having a low to intermediate risk for coronary artery disease, $n=16$ ), and patients with acute chest pain and intermediate risk but no electrocardiography changes and no biomarker evidence of an acute coronary syndrome $(n=23)$. Exclusion criteria for CT were: renal dysfunction (serum creatinine level $>1.5 \mathrm{mg} / \mathrm{dl}$ ), known hypersensitivity to iodinated contrast agent, and pregnancy. In addition, patients with cardiac valvular disease (including bicuspid aortic valves), with Marfan or Ehlers-Danlos syndrome, and patients that underwent previous intervention or operation on the heart, thoracic aorta, mediastinum, thoracic cage, and lung were excluded from this study. The institutional review board approved the study; written informed consent was waived since this was a retrospective analysis with no patient identification data included.

\section{CT data acquisition}

All patients underwent imaging with a dual source CT scanner (Somatom Definition, Siemens Medical Solutions, Forchheim, Germany) for clinical care reasons (i.e., exclusion of coronary artery disease) [1]. Details of scan protocol and data acquisition parameters can be found in [17]. ECGpulsing for radiation dose reduction was used in all patients [13]. Radiation dose associated with such a CT protocol is approximately 7-9 $\mathrm{mSv}[18]$.

\section{CT data analysis}

All measurements at CT were taken as described above for the cadaveric specimens (Fig. 1b) using standard cardiac post-processing software (Card IQ, Advantage Workstation 
4.0; GE Medical Systems, Milwaukee, Wis, USA). The left coronary ostium (LCO) was located on an oblique coronal multiplanar reconstruction, (MPR) which was orientated orthogonally to the plane of the aortic annulus. Correspondingly, the localization of the right coronary ostium (RCO) was performed on an oblique sagittal MPR. Measurements were taken from the base of the aortic annulus (at the junction of the sinus of Valsalva and the aortic cusps) to the center of the coronary ostia using an electronic calliper.

The height of the left coronary sinus (LS) was defined as the most distal attachment of the sinus to the aortic root and determined by moving through MPR parallel to the previously used coronal oblique view. Similarly, the height of the right coronary sinus was visualized on parallel sagittal oblique MPR. Again, all height measurements of the sinus were taken orthogonally to the plane of the aortic annulus.

The widths of the aortic root such as the aortic annulus (AA), the sinus of Valsalva (SV), and the sino-tubular junction (STJ) were measured on the same oblique coronal MPR as used for the localization of the left coronary ostium.

The inter-observer and intra-observer variation (J.K. and P.S.) was calculated for the first $20 \mathrm{CT}$ data sets for all measured parameters, with a time span of 3 months in between the measurements for both inter- and intraobserver variation. Both intra-observer variation (J.K.) (Right coronary ostium $(\mathrm{RCO})=0.65 \pm 1.45 \mathrm{~mm}$, left coronary ostium $(\mathrm{LCO})=0.37 \pm 1.27 \mathrm{~mm}$, annulus diameter $(\mathrm{AD})=1.41 \pm 1.22 \mathrm{~mm}$, right sinus height $(\mathrm{RS})=0.51 \pm$ $1.14 \mathrm{~mm}$, left sinus height (LS) $=0.17 \pm 0.97 \mathrm{~mm}$ ) and inter-observer variation $(\mathrm{RCO}=0.57 \pm 0.92 \mathrm{~mm}, \mathrm{LCO}=$ $0.62 \pm 0.78 \mathrm{~mm}, \quad \mathrm{AD}=1.46 \pm 1.11 \mathrm{~mm}, \quad \mathrm{RS}=0.27 \pm$ $0.9 \mathrm{~mm}, \mathrm{LS}=0.97 \pm 1.32 \mathrm{~mm}$ ) were low. Thus, the measurements in the remaining 130 patients were performed by only one reader (J.K.), being the same who made the measurements in the cadaver hearts.

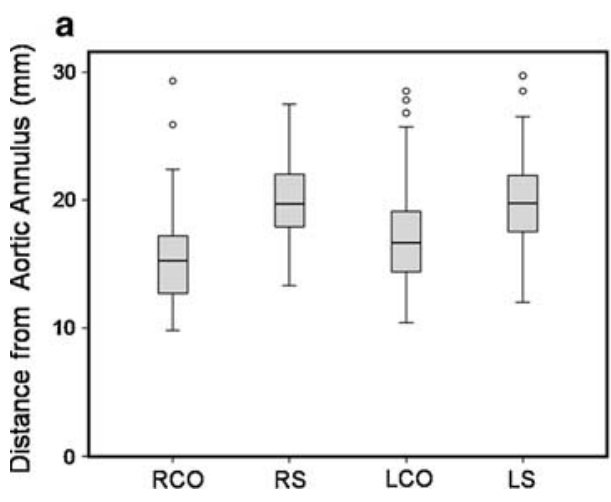

Fig. 2 CT coronary ostial and sinus measurements (a) Data from CT measurements showing right coronary ostial (RCO) and left coronary ostial (LCO) heights of $17.0( \pm 3.6)$ and $15.3( \pm 3.1) \mathrm{mm}$, respectively. The right (RS) and left (LS) sinus of Valsalva heights had mean values
Statistical analysis

Numerical values were expressed as frequencies or percentages. Age, BMI, BSA, heart rate, ostial locations (i.e., LCO and RCO), sinus heights (i.e., LS and RS), widths of the aortic root (i.e., AA, SW, and STJ), each represented normal distributions for both CT and cadaver measurements as evidenced by Kolmogorov-Smirnov test, and were presented as means \pm SD.

Mean differences between the left and right coronary ostium heights as well as sinus heights were assessed using paired $t$-tests. Differences of CT measures between male and female patients with respect to the LCO, RCO, LS, RS, were assessed using unpaired $t$-tests. Comparisons within the individual groups (CT and cadaver) of the RCO and LCO were performed using paired $t$-test.

The dependence of measurements on age, BSI, and BMA were investigated using Pearsons correlation. A P value of less than 0.05 was considered significant. Statistical analysis was performed using SPSS software (release 15.0, SPSS Inc., IL, USA).

\section{Results}

The dual-source CT examinations were successfully performed in all 150 patients and no complications occurred. All patients were in a sinus rhythm with a mean heart rate during scanning of $68 \pm 11 \mathrm{bpm}$ (range 54-103 bpm). Measurements could be made in all $150 \mathrm{CT}$ data sets and in all 75 human cadaveric specimens (Fig. 2, Table 3).

Ex vivo cadaver data

Mean RCO locations in relation to the aortic annulus in the cadaver hearts were $14.9( \pm 4.3) \mathrm{mm}[5-24 \mathrm{~mm}]$. Mean

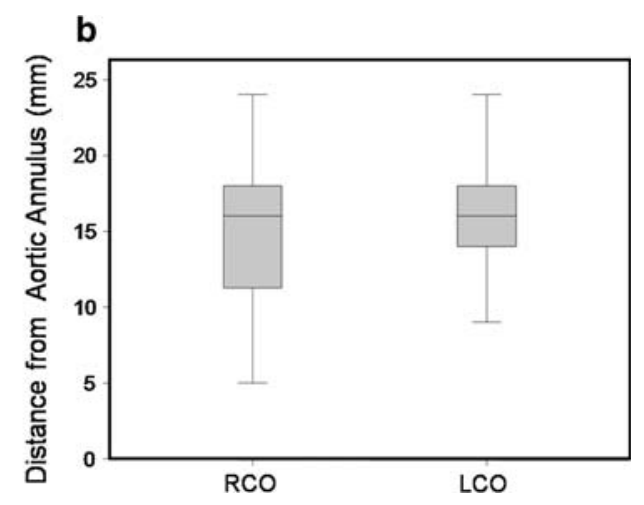

of $19.9( \pm 3.2) \mathrm{mm}$ and $19.8( \pm 2.7) \mathrm{mm}$, respectively. Cadaver Coronary Ostial (b) Data from the cadaver measurements showing right coronary ostial (RCO) and left coronary ostial (LCO) heights of 16.0 $( \pm 3.6)$ and $14.9( \pm 4.3) \mathrm{mm}$, respectively 
Table 3 Current study: coronary ostial location measurements for in vivo and ex vivo data

\begin{tabular}{|c|c|c|c|c|c|c|c|c|}
\hline Hearts & $\#$ & Sex & Age range & Mean age & General & Right (mm) & Left (mm) & $\begin{array}{l}\text { Annulus } \\
\text { diameter (mm) }\end{array}$ \\
\hline Cadaver & 75 & - & - & - & $\begin{array}{l}\text { All patients were greater } \\
\text { than } 40 \text { years of age }\end{array}$ & $14.9 \pm 4.3$ & $16.0 \pm 3.6$ & - \\
\hline CT & 150 & $61 \%$ M 39\% F & 29-86 years & 60.4 years & $\begin{array}{l}\% \text { of left ostium on } \\
\text { left sinus } 77.0 \% \text { of right } \\
\text { ostium on right sinus } 85.8\end{array}$ & $\begin{array}{l}\text { Ostium } 17.0 \pm 3.6 \\
\text { Sinus } 19.9 \pm 3.2\end{array}$ & $\begin{array}{l}\text { Ostium } 15.3 \pm 3.1 \\
\quad \text { Sinus } 19.8 \pm 2.7\end{array}$ & $23.0 \pm 3.8$ \\
\hline
\end{tabular}

$M=$ Male, $F=$ Female

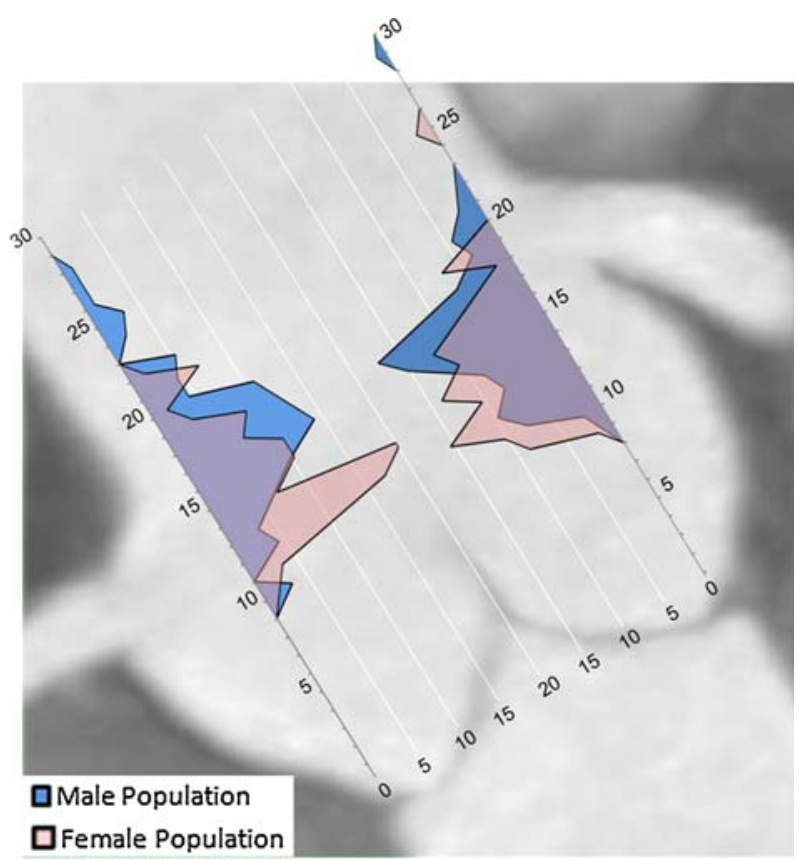

Fig. 3 Coronary ostial distribution-Visual representation of the distribution of the locations of the right and left coronary ostia overlaid onto a CT image showing the corresponding anatomy of the aortic root. Note the lower location of the right coronary artery (RCA) ostium and wider variation as compared to the ostium of the left main artery (LMA)

LCO showed a statistically significant difference at 16.0 $( \pm 3.6) \mathrm{mm}[9-24 \mathrm{~mm}](P<0.05)$.

\section{In vivo CT data}

CT data sets of the patients demonstrated a mean location above the aortic annulus of $17.0( \pm 3.6) \mathrm{mm}$ [10.4$28.5 \mathrm{~mm}$ ] for the RCO and a mean of $15.3( \pm 3.1) \mathrm{mm}$ [9.8$29.3 \mathrm{~mm}$ ] for the LCO (Fig. 3), with significant differences between the two sides $(P<0.0001)$. The mean height of the sinus of Valsalva was $19.9( \pm 3.2) \mathrm{mm}$ and $19.8( \pm 2.7) \mathrm{mm}$ for the RS and LS, respectively, with no significant difference between the two sides $(P=0.86)$. The RCO as a percentage of total RS height was $86 \%( \pm 13)$ [60-150\%] and the RCO as a percentage of total LS height was $77 \%$ $( \pm 12 \%)[51-126 \%](P<0.0001)$.
In males (Table 4), RCO and LCO were located at a mean of $18.0( \pm 3.7) \mathrm{mm} \quad(10.4-28.5 \mathrm{~mm})$ and 15.9 $( \pm 3.1) \mathrm{mm}(9.9-29.3 \mathrm{~mm})$ above the aortic annulus. In females, RCO and LCO were located at a mean of 15.5 $( \pm 2.9) \mathrm{mm}(10.9-22.0 \mathrm{~mm})$ and $14.5( \pm 3.0) \mathrm{mm}(9.8-$ $25.9 \mathrm{~mm}$ ) above the aortic annulus. There were significant differences between the genders for both ostial locations ( $P<0.0001$ for right and $P<0.01$ for left).

For males, the mean sinus of Valsalva heights of the RS and LS, respectively, were $20.9( \pm 3.2) \mathrm{mm}(13.0$ $29.7 \mathrm{~mm})$ and $20.9( \pm 2.5) \mathrm{mm}(14.4-27.5 \mathrm{~mm})$, and 18.3 $( \pm 2.6) \mathrm{mm}(12.0-24.4 \mathrm{~mm})$ and $18.2( \pm 2.2) \mathrm{mm}(13.3-$ $23.2 \mathrm{~mm})$ for females. There were significant differences between the genders for sinus heights $(P<0.0001)$.

To normalize the ostial locations for male and female, the coronary ostial locations were assessed as a percentage of the total sinus of Valsalva height, the mean RCO and LCO $86.2 \%( \pm 13.5 \%)$ and $75.8 \%( \pm 10.8 \%)$, respectively, for males, and $85.1 \%( \pm 11.7 \%)$ and $79.3 \%( \pm 12.3 \%)$, respectively, for females $(P=0.61$ for RCO as a percentage of total sinus height; and $P=0.073$ for LCO as a percentage of total sinus height).

The mean AA diameter was $24.5( \pm 2.7) \mathrm{mm}[18.1-$ $29.6 \mathrm{~mm}$ ] for males and $21.4( \pm 2.4) \mathrm{mm}[16.2-28.1 \mathrm{~mm}]$ for females $(P<0.0001)$.

In regards to all measurements (RCO, RS, LCO, LS, AA, SW, STJ), no dependence on age $(P>0.05)$ was found. Similarly, no correlation was found between BMI and RCO or LCO. However, measures of the LS $(P<0.01)$, RS $(P<0.05)$, AA $(P<0.05)$, SW $(P<0.05)$, STJ $(P<0.05)$ did show a significant correlation to BMI. In regards to BSA, a significant correlation $(P<0.05)$ was present for all measurements (RCO, RS, LCO, LS, AA, SW, STJ).

Comparison between in vivo and ex vivo data

When comparing the in vivo to the ex vivo measurements, little difference was found in the mean values for the left $(\mathrm{CT}=15.3 \mathrm{~mm}, \quad$ cadaver $=16.0 \mathrm{~mm}) \quad$ coronary ostium $(P=0.1675)$. A slightly larger, though statistically significant, difference was found for the mean right 
Table 4 Comparison of CT measurements by gender

\begin{tabular}{lllll}
\hline & & Male & Female & \\
\hline RCO & Mean & $18.0( \pm 3.7)$ & $15.5( \pm 2.9)$ & $P \leq 0.0001$ \\
& Range & $10.4-28.5$ & $10.9-22.0$ & \\
LCO & Mean & $15.9( \pm 3.1)$ & $14.5( \pm 3.07)$ & $P \leq 0.0001$ \\
& Range & $9.9-29.3$ & $9.8-25.9$ & \\
RS & Mean & $20.9( \pm 3.2)$ & $18.3( \pm 2.6)$ & \\
& Range & $13.0-29.7$ & $12.0-24.4$ & $P<0.0001$ \\
LS & Mean & $20.9( \pm 2.5)$ & $18.2( \pm 2.2)$ & \\
& Range & $14.4-27.5$ & $13.3-23.2$ & $P=0.61$ \\
$\%$ of RCO on RS & Mean & $86.2 \%( \pm 13.5 \%)$ & $85.1 \%( \pm 11.7 \%)$ & $P=0.073$ \\
Annulus diameter & Mean & $75.8 \%( \pm 10.8 \%)$ & $79.3 \%( \pm 12.3 \%)$ & $P<0.0001$ \\
& Mean & $24.0( \pm 4.2)$ & $21.4( \pm 2.4)$ & \\
\hline
\end{tabular}

resin under pressure would likely lower the differences found in the in vivo and ex vivo results) in the development and implementation strategies for devices, techniques, and treatment protocols associated with the aortic root and coronary arteries, as cadaver measurements may not give an accurate representation of the in vivo setting.

\section{Comparison to previous studies}

Our measurements of left and right sinus of Valsalva height have general agreement with previous studies $[3,19,20]$.

Because of the various methods of measurements used to assess coronary ostial location in previous cadaveric studies, direct comparisons are challenging. The main points of comparison to the current study are given in Table 1.

Jatene et al. [12] measured the distance from the bottom of the sinus to the corresponding coronary ostium along the surface of the sinus in 100 cadavers. This method should give measurements higher than obtained with the procedure used in our study. However, mean ostial locations of $14.8 \mathrm{~mm}$ and $13.3 \mathrm{~mm}$ for right and left coronary origins (standard deviations not given) were found. Our cadaver measurements give slightly higher values for right and left ostial origins, even though we measure a shorter (straighter) distance. Jatene et al. also separated their data into age groups of below and above 40 years of age, which gave different mean ostial locations for the different age groups ( $<40 \mathrm{y} / \mathrm{o} ; 14.6 \mathrm{~mm}$ and $13.0 \mathrm{~mm}$ for right and left ostium, respectively, and $>40 \mathrm{y} / \mathrm{o} ; 16.1 \mathrm{~mm}$ and $14.9 \mathrm{~mm}$ for right and left ostium, respectively). The ostial locations given by Jatene et al. in the over 40-year age group are similar to this study.

Cavalcanti et al. [6] and Vlodaver et al. [22] gave results of their coronary ostial measurements with respect to the intercommissural line (defined in each of their manuscripts through a figure as a straight line from the two attachment points of the cusps to the sinus of Valsalva) in cadaveric 
hearts. It is difficult to compare our data directly to these studies, as we used the aortic annulus, which correlates to the inferior limit of the sinus, as a reference instead of the intercommissural line.

Two previous studies (by Turner and Navaratnam [21] and Muriago et al. [15]) used the sinotubular junction as the reference for measurements of the coronary ostia. We chose not to re-evaluate the data relative to this position as it is our feeling that the aortic annulus is a more consistent and reproducible reference point, and is more readily viewable using clinical imaging modalities. Further, for insertion of a PAV, the aortic annulus is the hinge point of the overstented aortic valve and is the more relevant reference in relation to the coronary ostia.

\section{Clinical implications}

Concerning percutaneous and transapical valve replacement, obstruction of the coronary arteries during and/or after implantation remains a risk that can have catastrophic consequences. Boudjemline and Bonhoeffer [4] point out that precise placement, with respect to height of the PAV, is crucial. Locations too high above the valve annulus result in coronary ostial obstruction and locations too low can negatively impact left ventricular and/or mitral valve function. The large distribution of ostial locations found in this study, emphasize the importance of considering such variations in the development of treatments.

\section{Limitations}

This study was performed from a patient group consisting of Caucasian male and female patients, and hence does not take into account possible differences in ostial location within different ethnic groups. The study is also limited by inter- and intra-observer variability being performed only for the CT patients group and not for the cadaveric group. Further no data on age or sex was available for the cadaver measurements. Planes used for the measurements made in CT may not be exactly the same as those used in the anatomic specimens.

\section{Conclusions}

This study provides values representing the normal distribution of coronary ostial locations relative to the aortic root in patients and in cadaver specimens. CT showed the right and left ostia to be located in the upper quarter of their respective sinus of Valsalva. The significant differences found between the in vivo and ex vivo data strengthen the call for in vivo measurements, or cadaveric measurement techniques that reproduce the in vivo environment, to be used in the planning of interventional or surgical devices and procedures involving the coronary ostia. In addition, the large variations found in both right $(10.4-28.5 \mathrm{~mm})$ and left $(9.8-29.3 \mathrm{~mm})$ coronary ostial locations are an interesting finding that point to the importance of considering such variations in the development of treatments. Future studies must aim at an assessment of coronary ostial and aortic root anatomy in patients suffering from severe aortic stenosis being candidates to undergo percutaneous or transapical valve replacement.

Acknowledgments This paper was supported by the National Center of Competence in Research for Computer Aided and Image Guided Medical Interventions (NCCR CO-ME) of the Swiss National Science Foundation.

\section{References}

1. Alkadhi H, Scheffel H, Desbiolles L, Gaemperli O, Stolzmann P, Plass A, Goerres GW, Luescher TF, Genoni M, Marincek B, Kaufmann PA, Leschka S (2008) Dual-source computed tomography coronary angiography: influence of obesity, calcium load, and heart rate on diagnostic accuracy. Eur Heart J 29(6):766-776

2. Babaliaros V, Block P (2007) State of the art percutaneous intervention for the treatment of valvular heart disease: a review of the current technologies and ongoing research in the field of percutaneous valve replacement and repair. Cardiology 107(2):87-96

3. Berdajs D, Lajos P, Turina M (2002) The anatomy of the aortic root. Cardiovasc Surg 10(4):320-327

4. Boudjemline Y, Bonhoeffer P (2002) Steps toward percutaneous aortic valve replacement. Circulation 105(6):775-778

5. Budoff MJ, Achenbach S, Blumenthal RS, Carr JJ, Goldin JG, Greenland P, Guerci AD, Lima JA, Rader DJ, Rubin GD, Shaw LJ, Wiegers SE (2006) Assessment of coronary artery disease by cardiac computed tomography: a scientific statement from the American Heart Association Committee on Cardiovascular Imaging and Intervention, Council on Cardiovascular Radiology and Intervention, and Committee on Cardiac Imaging, Council on Clinical Cardiology. Circulation 114(16):1761-1791

6. Cavalcanti JS, de Melo NC, de Vasconcelos RS (2003) Morphometric and topographic study of coronary ostia. Arq Bras Cardiol 81(4):359-362, 355-358

7. Diamond GA, Forrester JS (1979) Analysis of probability as an aid in the clinical diagnosis of coronary-artery disease. $\mathrm{N}$ Engl J Med 300(24):1350-1358

8. Du Bois D, Du Bois EF (1989) A formula to estimate the approximate surface area if height, weight be known 1916. Nutrition 5(5):303-311 (discussion 312-313)

9. Flecher EM, Curry JW, Joudinaud TM, Kegel CL, Weber PA, Duran CM (2007) Coronary flow obstruction in percutaneous aortic valve replacement an in vitro study. Eur J Cardiothorac Surg 32(2):291-294

10. Hendel RC, Bateman TM, Cerqueira MD, Iskandrian AE, Leppo JA, Blackburn B, Mahmarian JJ (2005) Initial clinical experience with regadenoson, a novel selective A2A agonist for pharmacologic stress single-photon emission computed tomography myocardial perfusion imaging. J Am Coll Cardiol 46(11):2069-2075

11. Huber CH, Tozzi P, Corno AF, Marty B, Ruchat P, Gersbach P, Nasratulla M, von Segesser LK (2004) Do valved stents compromise coronary flow? Eur J Cardiothorac Surg 25(5):754-759 
12. Jatene MB, Monteiro R, Guimaraes MH, Veronezi SC, Koike MK, Jatene FB, Jatene AD (1999) Aortic valve assessment. Anatomical study of 100 healthy human hearts. Arq Bras Cardiol 73(1):75-86

13. Leschka S, Scheffel H, Desbiolles L, Plass A, Gaemperli O, Valenta I, Husmann L, Flohr TG, Genoni M, Marincek B, Kaufmann PA, Alkadhi H (2007) Image quality and reconstruction intervals of dual-source CT coronary angiography: recommendations for ECG-pulsing windowing. Invest Radiol 42(8):543-549

14. Lutter G, Ardehali R, Cremer J, Bonhoeffer P (2004) Percutaneous valve replacement: current state and future prospects. Ann Thorac Surg 78(6):2199-2206

15. Muriago M, Sheppard MN, Ho SY, Anderson RH (1997) Location of the coronary arterial orifices in the normal heart. Clin Anat 10(5):297-302

16. Scheffel H, Alkadhi H, Plass A, Vachenauer R, Desbiolles L, Gaemperli O, Schepis T, Frauenfelder T, Schertler T, Husmann L, Grunenfelder J, Genoni M, Kaufmann PA, Marincek B, Leschka S (2006) Accuracy of dual-source CT coronary angiography: First experience in a high pre-test probability population without heart rate control. Eur Radiol 16(12):2739-2747
17. Stolzmann P, Scheffel H, Schertler T, Frauenfelder T, Leschka S, Husmann L, Flohr TG, Marincek B, Kaufmann PA, Alkadhi H (2007) Radiation dose estimates in dual-source computed tomography coronary angiography. Eur Radiol 18:592-599

18. Stolzmann P, Scheffel H, Schertler T, Frauenfelder T, Leschka S, Husmann L, Flohr TG, Marincek B, Kaufmann PA, Alkadhi H (2008) Radiation dose estimates in dual-source computed tomography coronary angiography. Eur Radiol 18(3):592-599

19. Swanson M, Clark RE (1974) Dimensions and geometric relationships of the human aortic valve as a function of pressure. Circ Res 35(6):871-882

20. Thubrikar M (1990) The aoric valve. CRC Press, Inc, Boca Raton, Florida, USA

21. Turner K, Navaratnam V (1996) The positions of coronary arterial ostia. Clin Anat 9(6):376-380

22. Vlodver Z, Vlodaver Neufeld HN, Edward JE (1975) Coronary arterial variations in the normal heart and in congential heart disease. Academic Press Inc, New York 